\title{
A Cross-Layered Path Stability Based Routing Protocol for WiMAX Networks
}

\author{
${ }^{1}$ Deva Priya, M. and ${ }^{2}$ M.L. Valarmathi \\ ${ }^{1}$ Department of CSE, Sri Krishna College of Technology, Coimbatore, Tamil Nadu, India \\ ${ }^{2}$ Department of CSE, Government College of Technology, Coimbatore, Tamil Nadu, India
}

Received 2013-05-26, Revised 2013-09-02; Accepted 2013-09-09

\begin{abstract}
In IEEE 802.16, a Subscriber Station (SS) is battery powered with limited capacity. A SS can be made to operate for longer durations by deploying power saving features or by using relay stations. Multi-hop communication conserves power, but finding an optimal path to the destination is monotonous. Path stability indicates how stable a path is and how long it can support communication. Identifying stable paths helps in reducing the control traffic, number of connection disruptions and also in conserving power. In this study, Greedy Perimeter Stateless Routing (GPSR) and Link-St Ability and Energy aware Routing (LAER) designed for Mobile Ad hoc NET works (MANETs) are implemented for WiMAX networks and their performance is evaluated. Further, a novel Cross-layered Path Stability based Routing protocol (CPSR) is proposed to find a stable path based on Residual Energy (RE), Received Signal Strength Indicator (RSSI) and Signal-to-Noise Ratio (SNR). The total energy consumed is minimized, thus maximizing the network lifetime.
\end{abstract}

Keywords: WiMAX, Quality of Service (QoS), Residual Energy, Received Signal Strength Indicator, Signal-to-Noise Ratio, GPSR

\section{INTRODUCTION}

WiMAX provides broadband wireless last-mile access in a Metropolitan Area Network (MAN) with performance comparable to traditional cables. It is one of the major competing mesh technologies and has attracted significant interests recently.

The fastest Wi-Fi connection can transmit up to 54 megabits per second under optimal conditions, while WiMAX can handle up to 70 megabits per second.

IEEE 802.16 operates either in cellular-like PMP (Point-to-Multipoint) mode or Mesh mode. Two layers defined in WiMAX are the PHY and the Medium Access Control (MAC) layers. Itoffers a transmission range of few kms and guarantees Quality of Service (QoS) by supporting various service classes and providing sufficient bandwidth. Different types of service classes supported by IEEE 802.16 are classified into one of the following: (1) Unsolicited Grant Service (UGS) (2) Realtime Polling Service (rtPS) (3) Non-Real-time Polling Service (nrtPS) and (4) Best Effort (BE) Service.
Operation at all levels of the communication protocol stack has an impact on energy consumption and hence it becomes mandatory to address energy efficiency jointly in the device, the PHY layer, the MAClayer and the network layer.

In this study, a stable energy based routing protocol is designed for mesh mode WiMAX networks. In a mesh network involving multiple hops, the next hop node is selected based on the Residual Energy (RE) of the node, so as to avoid early depletion of energy in the network. In addition, the Received Signal Strength Indicator (RSSI) and the Signal-to-Noise Ratio (SNR) are also taken into consideration.

\section{RELATED WORK}

Various routing mechanisms are proposed in the literature for mesh mode WiMAX networks.

Some routing algorithms select paths with minimum total transmission power. If the links are assumed to be error-free, there is no need for retransmission; energyCorresponding Author: Deva Priya, M., Department of CSE, Sri Krishna College of Technology, Coimbatore, Tamil Nadu, India 
efficient routing algorithms choose the minimum-hop paths (Chen and Gursoy, 2009).

Spectral reuse in resource allocation for IEEE 802.16 mesh networks along with Routing Tree Construction (RTC), bandwidth allocation, time-slot assignment and bandwidth guarantee of real-time flows is proposed in (Chen et al., 2009).

A joint routing and scheduling mechanism known as Maximum Spatial Reuse (MSR) designed with an objective of minimizing the length of a schedule and maximizing the spectrum spatial reuse without violating the Signal-to-Interference Noise Ratio (SINR) is proposed in (El-Najjar et al., 2010).

Amin et al. (2007) has presented an integrated routing and link scheduling schemefor persistent communication of fast moving vehicles.

A joint routing and scheduling algorithm which selects the highest end-to-end data rate paths for maximizing network capacityis proposed in (Nahle and Malouch, 2008).

Kim et al. (2008), authors have proposed a proactive hop-by-hop QoS based routing protocol.

Routing algorithms proposed by various authors are reviewed in (Qassem et al., 2009).

Multipath routing for efficient utilization of wireless radio resources by providing spectral efficiency and least mean path interference for multiple hops is studied in (Kelaiya, 2008). In addition, a scheduling algorithm is also designed to find the maximum number of concurrent transmissions that satisfies the Signal-toInterference Noise Ratio (SINR) constraints.

A routing algorithm for coordinated distributed scheduling called Better Approach to Mobile Ad hoc Networking (BATMAN) is proposed in (Ntlatlapa, 2008). It does not maintain the full route to the destination, but the information about the next link that yields the best route.

In coordinated distributed scheduling, contentionfree slots in the control sub frame are allocated to mesh radios for sending and receiving bandwidth change requests and responses respectively. A dynamic scheme based on classification that permits traffic to be routed directly between SSs is proposed in (Zhang et al., 2009).

Kuran et al. (2008), authors have constructed a routing tree by considering only the first parent node in the tree.

A routing algorithm incorporated in the MAC layer is proposed in ( $\mathrm{Li}$ et al., 2009). To avoid being trapped in path loops, a loop cancelation mechanism is included and each node determines the next-hop nodes and attempts to forward packets in the earliest slots.
A Routing and Call Admission Control (CAC) mechanism based on Shortest Widest Effective Bandwidth (SWEB) is proposed in (Tsai and Wang, 2007) considering parameters like packet error rate, capacity over the link and the hop count from the source to the destination.

Zhang et al. (2010), another joint CAC and routing scheme for multiple services is proposed to prioritize classes by imposing different reward rates. To obtain optimal CAC policies, optimization techniques are applied to decision processes.

Al-Hemyari et al. (2008), authors have proposed arouting and collision free Centralized Scheduling (CS) algorithm involving cross layers-the Network and the MAC layer.

Jalali and Khorsandi (2011), a routing tree is constructed dynamically and a centralized scheduling algorithm is designed based on the feedback from the network. The Scheduling algorithm involves the MAC and the Network layer.

Cross-layer optimization and computing of transport capacity dedicated to Internet access is proposed in (Molle et al., 2008).

Jiao et al. (2010), a cross-layer concurrent transmission scheduling algorithm that uses a scheduling strategy to activate available potential multiple links and enhance concurrent transmission is proposed. An adaptive power control scheme is designed inthe physical layer tosupport different transmission rates for different paths and to regulate link transmission rates at different layers based on the traffic loads.

Mogre et al. (2008), authors have proposed Centrally Optimized Routing Extensions (CORE) to address the issues of optimized routing, scheduling and bandwidth allocationby coding the network and increasing the number of admitted flows.

Peng and Sun (2011), a novel data scheduling scheme is proposed based on an optimal model of mini-slot allocation so as to reduce the number of mini-slot segments, keeping continuous mini-slots as long as possible.

Lo and Ou (2009), routing and centralized scheduling algorithms are proposed to construct a routing tree from the given mesh topology and to allocate channel mini-slots.

A cross-layer design architecture in which transmission power control, route construction and centralized scheduling are taken into account is presented in (Peng et al., 2007). Interference and hopcount are jointly considered to construct the routing 
path. The neighbor degree metric, a function oftransmission power and number of neighbor nodesis used to construct the routing tree.

In contrast to the routing algorithms that use shortest paths and transitive reachability to find routes, Greedy Perimeter Stateless Routing (GPSR) proposed by (Karp and Kung, 2000) uses the positions and connectivity of nodes to make packet forwarding decisions. When the topology changes frequently, GPSR uses the local topology information to find new routes.

The stability of a link is given by its probability to persist for a certain time span, which is not necessarily linked with its probability to reach a very high age. Linkstability and Energy aware Routing protocols (LAER) requires each node to advertise its location, rate of energy consumption and link stability index for each outgoing link of node 'i' (De Rango et al., 2012). This next hop selection scheme minimizes the joint energy stability metric.

Previous works do not predict the movement of the relays out of the coverage area in a multi-hop network. Further, the routing algorithms discussed in the literature do not take the Residual Energy (RE), Received Signal Strength Indicator (RSSI) and Signal-to-Noise Ratio (SNR) together into consideration.

\section{PROPOSED SYSTEM}

Cross-layered Path Stability based Routing protocol (CPSR) finds a stable path based on all the three factorsRSSI, RE and SNR.

Links arechosen based on the energy reserve in the next hop nodes. By preserving the energy in the network, the lifetime of the network can be extended.

GPSR and LAER algorithms designed for MANETs are implemented for WiMAX networks. GPSR is based on the shortest distance to the destination while LAER is based on the Link Stability (LS) and the Mean Drain Rate (MDR).

GPSR is modified so as to make it appropriate for WiMAX networks. Cross layer integration is done in WiMAX 802.16.cc file by setting the packet type as GPSR, thus enabling GPSR in WiMAX.

Cross layering involves the Network layer, MAC layer and PHY layer. Routing in the Network Layer is performed using GPSR. Multi-hop relay communication is enabled by MAC $802.16 \mathrm{j}$. Both the PHY layer and the Network layer are integrated. The MAC and the Network layers monitor the PHY layer to determine a stable path based on the parameters obtained from the PHY layer.

LAER chooses the next hop nodes based on the stability of links. It does not find stable paths. Hence to overcome this shortcoming, CPSR is proposed to find a stable path in a multi-hop network.

In CPSR, path request messages are forwarded to find paths to the destination. Each node stores the LS factor of its predecessors, appends its $\mathrm{LS}_{\mathrm{i}}$ and forwards it to its neighbors. This takes place in forward and reverse directions and both Forward Link Stability $\left(\mathrm{LS}_{\mathrm{F}}\right)$ and Reverse Link Stability $\left(\mathrm{LS}_{\mathrm{R}}\right)$ factors are calculated.

Thresholds are set for RE, RSSI and SNR and the nodes with values above the threshold are selected as next hop nodes.

RE should be $>30 \%$. As nodes with less energy are not selected as next hop nodes, energy isconserved to a certain extent. Early depletion of energy is avoided by choosing the paths dynamically.

The RSSI threshold denoted by 'Pre_GZ_Thresh' is used to detect Gray Zones. If the signal strength of a relay is high, then it is near. If it moves out of range, itsRSSI goes below threshold. Path advertisement packets from such relays should be discarded, so as to avoid unstable links during path discovery.

In LAER, the Stability of a node is calculated as shown in Equation (1). It is the reciprocal measure of stability (De Rango et al., 2012):

$\mathrm{S}_{\mathrm{i}, \mathrm{j}}=\frac{\mathrm{d}_{\mathrm{i}, \mathrm{j}}^{\mathrm{avg}}}{\mathrm{R}_{\mathrm{i}, \mathrm{j}}\left(\mathrm{a}_{\mathrm{i}, \mathrm{j}}\right) \cdot \mathrm{k}} \forall(\mathrm{i}, \mathrm{j})$

Where:

$\mathrm{d}_{\mathrm{i}, \mathrm{j}}=$ Euclidean distance between nodes ' $\mathrm{i}$ ' and ' $\mathrm{j}$ '

$\mathrm{R}_{\mathrm{i}, \mathrm{j}}=$ Stability index accounting for link duration in previous observation intervals

$\mathrm{a}_{\mathrm{i}, \mathrm{j}}=$ Age of the link between ' $\mathrm{i}$ ' and ' $\mathrm{j}$ '

$\mathrm{k}=$ Scaling factor

In LAER, a node with less Stability Factor $\mathrm{S}_{\mathrm{i}, \mathrm{j}}$ is selected. But in CPSR, a stable path is found by selecting the next hop node with high RE, RSSI and less Noise ratio (in SNR). In other words, initially a more stable link is selected and then a path with high stability is found.

In CPSR, Link Stability for a node is calculated as follows Equation (2): 
$\mathrm{s}_{\mathrm{i}, \mathrm{j}}=\frac{\left(\mathrm{RSSI}_{\mathrm{i}, \mathrm{j}}+\mathrm{RE}_{\mathrm{i}, \mathrm{j}}\right)-\left(\mathrm{RE}_{\mathrm{THRESH}}+\mathrm{RSSI}_{\mathrm{THRESH}}\right)}{\left(\mathrm{RE}_{\mathrm{THRESH}}+\mathrm{RSSI}_{\text {THRESH }}\right)}$

Where:

$$
\begin{aligned}
\operatorname{RSSI}_{\mathrm{i}, \mathrm{j}} \text { and RSSI } & \text { RHRESH }= \\
& \text { RSSI of link }(\mathrm{i}, \mathrm{j}) \text { and RSSI } \\
\mathrm{RE}_{\mathrm{i}, \mathrm{j}} \text { and } \mathrm{RE}_{\mathrm{THRESH}}= & \mathrm{RE} \text { of link }(\mathrm{i}, \mathrm{j}) \text { and } \mathrm{RE} \\
& \text { Threshold respectively }
\end{aligned}
$$

In CPSR, a node with high $\mathrm{S}_{\mathrm{i}, \mathrm{j}}$ is selected, contrary to LAER. $\mathrm{S}_{\mathrm{i}, \mathrm{j}}$ can never be negative as a node with values above threshold are selected.

The next hop node with less Noise Ratio is selected. In case if its Noise Ratio is above threshold, then the node with next higher value of $\mathrm{S}_{\mathrm{i}, \mathrm{j}}$ is selected, provided it satisfies the SNR threshold. If more than one node satisfies the constraints, then the tie is broken by random selection.

If nodeswith RSSI, RE and Noise Ratio (NR) values corresponding to the mentioned thresholdsdo not exist, then there will be no next hop node to receive the packets. In that case, the SNR value is not taken into consideration and the node with better RSSI and RE is selected (above threshold). Even after circumventing SNR, if RSSI and RE do not have the required (threshold) values, then the packet is not forwarded, but dropped.

Both Forward $\left(\mathrm{LS}_{\mathrm{F}}\right)$ and Reverse $\left(\mathrm{LS}_{\mathrm{R}}\right)$ stability factors are computed and the Average Path Stability (PS) is calculated as follows Equation (3-6):

$$
\mathrm{LS}_{\mathrm{F}}=\sum_{\mathrm{i}=1}^{\mathrm{n}} \mathrm{S}_{\mathrm{i}, \mathrm{j}}^{\mathrm{F}} \text { in Forward direction }
$$

$\mathrm{LS}_{\mathrm{R}}=\sum_{\mathrm{i}=1}^{\mathrm{n}} \mathrm{S}_{\mathrm{i}, \mathrm{j}}^{\mathrm{R}}$ in Re verse direction

$$
\text { Stability Factor }(\mathrm{SF})=\mathrm{LS}_{\mathrm{F}}+\mathrm{LS}_{\mathrm{R}}
$$

Average Path Stability $=\left(\mathrm{PS}_{\mathrm{AVG}}\right)=\frac{\mathrm{SF}}{\text { Hop_Count }}$

The stability of a path $\left(\mathrm{PS}_{\text {path }}\right)$ is based onAverage Path Stability $\left(\mathrm{PS}_{\mathrm{AVG}}\right)$ and the Standard Deviationof Stability factors. Standard Deviation (SD) is computed as shown in Equation (7). It is based on hop count, Average Path Stability ( $\mathrm{PS}_{\mathrm{AVG}}$ ) and the Forward and
Backward Stability factors $\left(S_{i, j}^{F}\right.$ and $\left.S_{i, j}^{R}\right)$ :

$$
\mathrm{SD}=\sqrt{\frac{\sum_{\mathrm{i}=1}^{\mathrm{n}}\left(\mathrm{S}_{\mathrm{i}, \mathrm{j}}^{\mathrm{F}}-\mathrm{PS}_{\mathrm{AVG}}\right)^{2}+\sum_{\mathrm{i}=1}^{\mathrm{n}}\left(\mathrm{S}_{\mathrm{i}, \mathrm{j}}^{\mathrm{R}}-\mathrm{PS}_{\mathrm{AVG}}\right)^{2}}{\text { Hop_Count }}}
$$

The stability of a path $\left(\mathrm{PS}_{\text {path }}\right)$ is given by Equation (8):

$\mathrm{PS}_{\mathrm{path}}=\frac{\mathrm{PS}_{\mathrm{AVG}}}{\mathrm{SD}}$

The path with maximum $\mathrm{PS}_{\text {path }}$ is selected for transmission from source ' $i$ ' to destination ' $j$ '. In other words, the stable path is the one with less number of hops.

\section{PERFORMANCE ANALYSIS}

The system is simulated using ns-2 and the performance is analyzed in terms of Packet Delivery Ratio (PDR), Throughput, Packet Loss Ratio (PLR), Routing Overhead and Energy consumed. The simulation parameters are listed in Table 1.

From the results it is evident that, CPSR outperforms GPSR and LAER. From Fig. 1, it is clear that CPSR yields a better Packet Delivery Ratio (PDR) when compared to GPSR and LAER. The Throughput of CPSR is high when compared to GPSR and LAER (Fig. 2).

Packet Loss Ratio (PLR) of CPSR is low, while that of GPSR is high (Fig. 3). CPSR involves the least delay (Fig. 4).

Table 1. Simulation parameters

\begin{tabular}{ll}
\hline Parameter & Value \\
\hline MAC protocol & $802.16 \mathrm{j}$ \\
Transmit power & $35 \mathrm{~mW}$ \\
Bandwidth & $50 \mathrm{Mbps}$ \\
Initial energy & $100 \mathrm{~J}$ \\
RSSI threshold & $-75 \mathrm{dBm}$ \\
Mobility model & Random-waypoint \\
Propagation & Two ray ground \\
Packet size & $1024 \mathrm{bits}$ \\
SNR threshold & $10 \mathrm{dBm}$ \\
Data rate & $2 \mathrm{Mbps}$ \\
Routing protocol & $\mathrm{GPSR}$ \\
Carrier frequency & $2.4 \mathrm{GHz}$ \\
Number of nodes & 100 \\
Transmission range & $250-400 \mathrm{~m}$ \\
Speed of nodes & $20 \mathrm{~ms}^{-1}$ \\
\hline
\end{tabular}


Deva Priya, M. and M.L. Valarmathi / American Journal of Applied Sciences 10 (11): 1325-1334, 2013

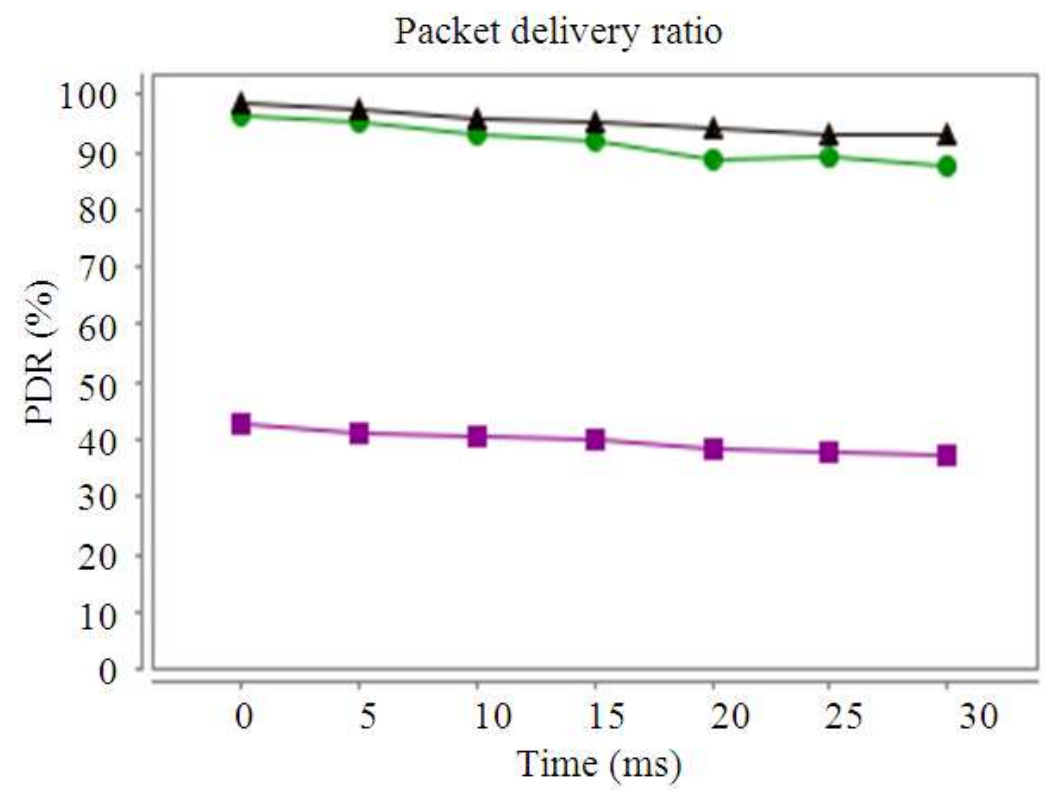

GPSR $\bullet-\mathrm{LAER} \star \mathrm{CPSR}$

Fig. 1. Packet delivery ratio

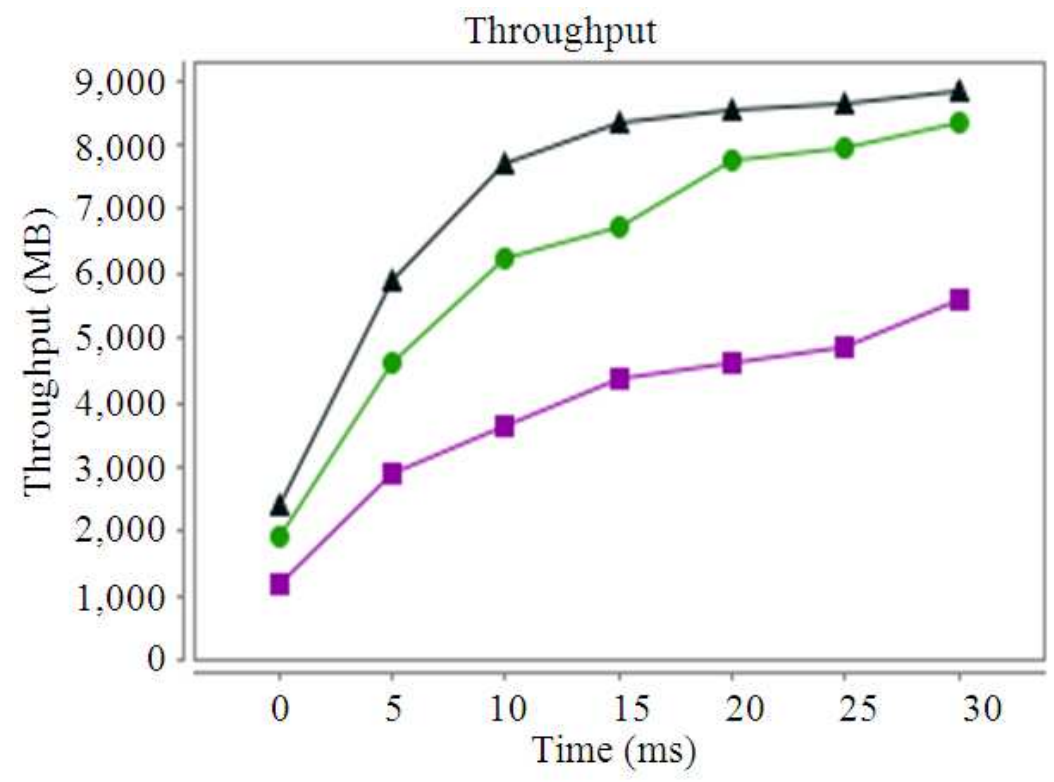

-GPSR $-\mathrm{LAER} ₫ \mathrm{CPSR}$

Fig. 2. Throughput 
Deva Priya, M. and M.L. Valarmathi / American Journal of Applied Sciences 10 (11): 1325-1334, 2013

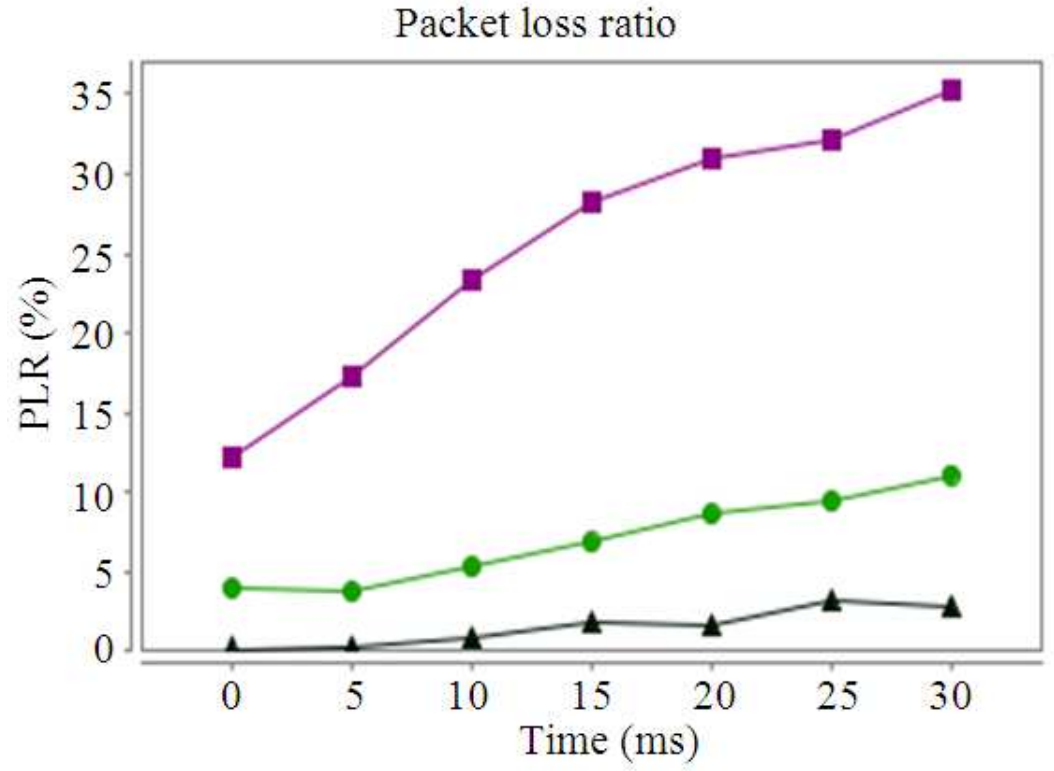

GPSR $\bullet-\mathrm{LAER} \_-\mathrm{CPSR}$

Fig. 3. Packet loss ratio

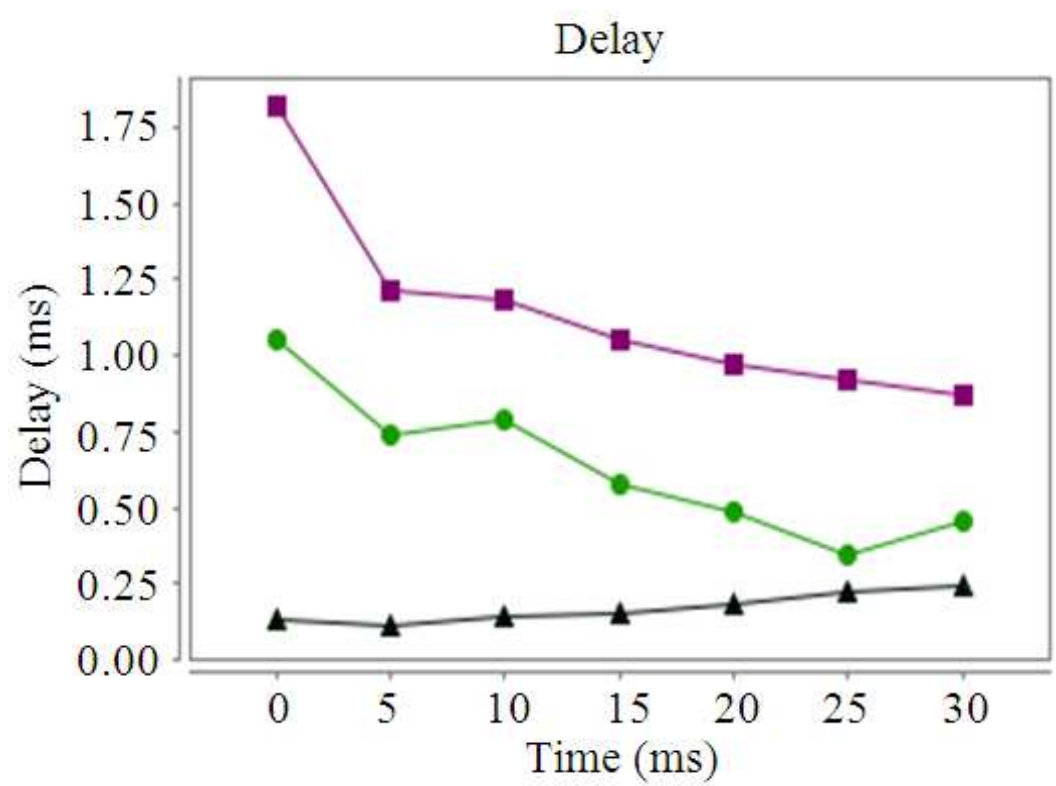

- $-\mathrm{GPSR} \rightarrow \mathrm{LAER} \star \mathrm{CPSR}$

Fig. 4. Delay 
Deva Priya, M. and M.L. Valarmathi / American Journal of Applied Sciences 10 (11): 1325-1334, 2013

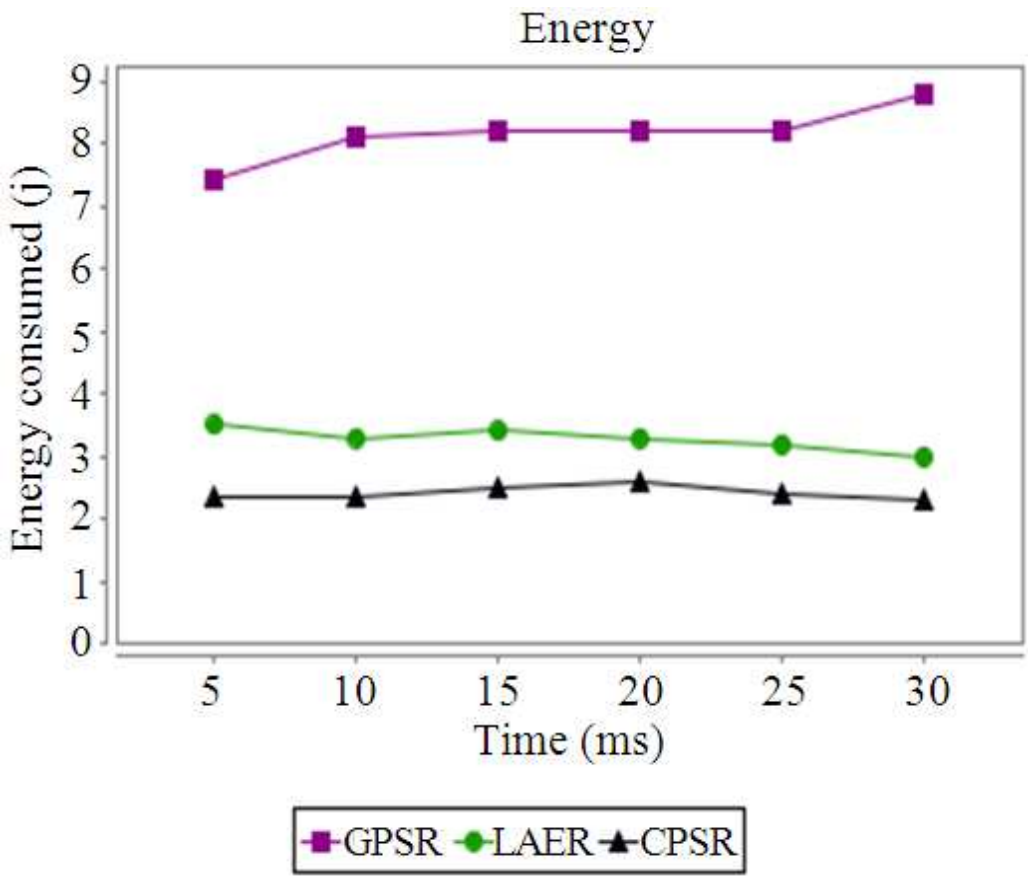

Fig. 5. Energy consumed

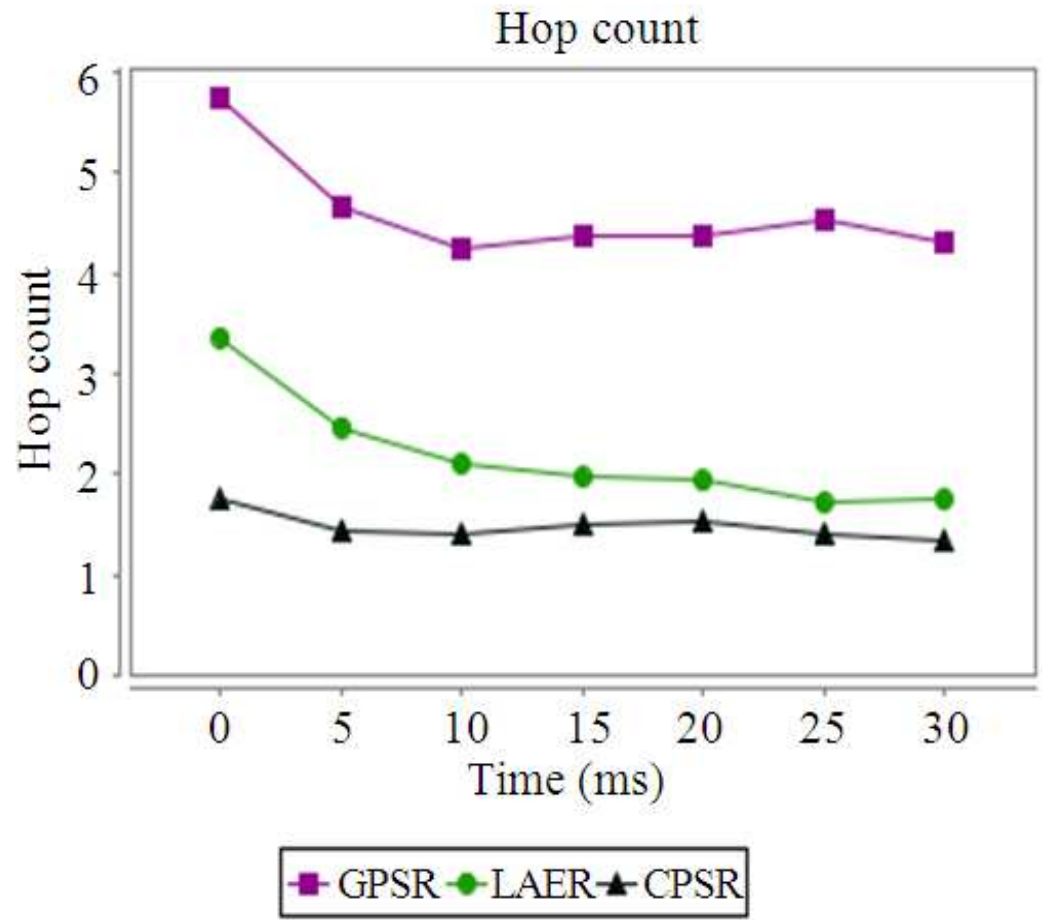

Fig. 6. Hop count 


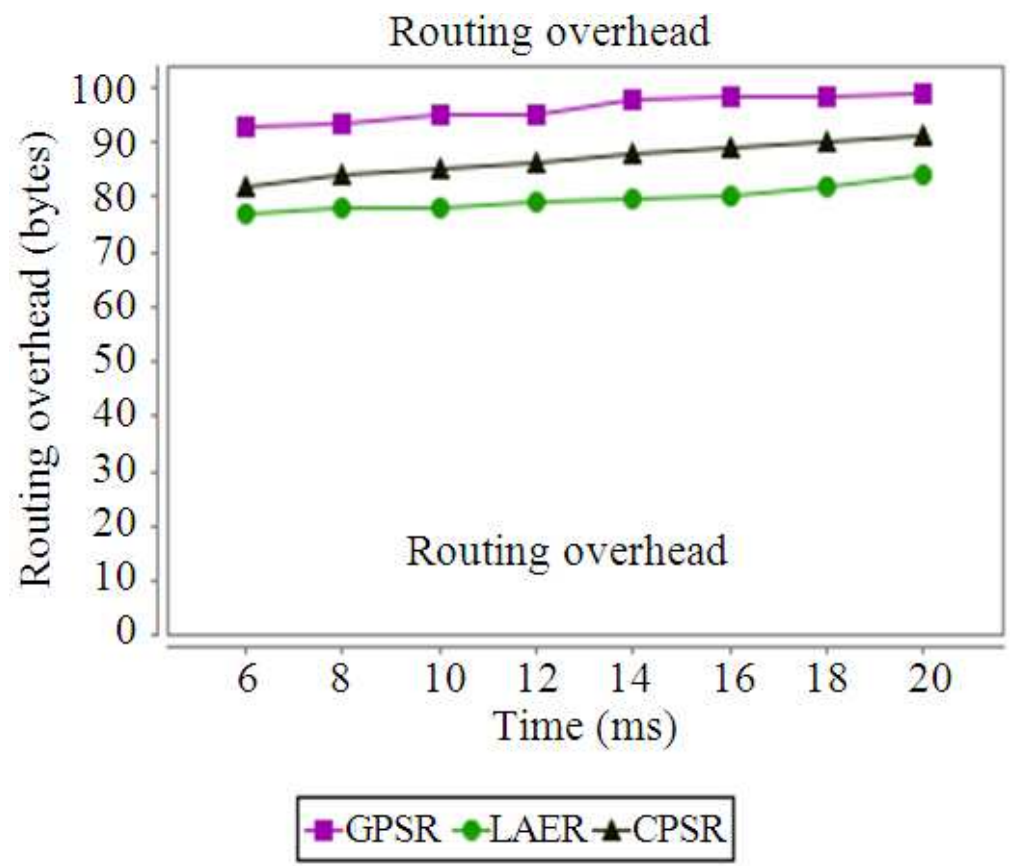

Fig. 7. Routing overhead

From Fig. 5, it is explicit that GPSR consumes more energy when compared to CPSR and LAER. CPSR consumes the least amount of energy, thus maximizing the lifetime of the network.

The stability of a path is based on the number of hops. The hop count gives the number of relays involved. CPSR involves the least number of hops (Fig. 6). As the number of relays involved is reduced, the total energy consumed is minimized.

During path establishment, the routing information involved in neighbor discovery constitutes the routing overhead. In CPSR, as the nodes are selected based on RSSI, SNR and RE, the routing overhead is reduced when compared to GPSR (Fig. 7).

\section{CONCLUSION}

The behavior of GPSR, LAER and CPSR are analyzed for a multi-hop WiMAX network. CPSR based on Residual Energy (RE), Received Signal Strength Indicator (RSSI) and Signal to Noise Ratio (SNR) ensures path stability.

A stable path is established between the source and the destination by selecting the next hop node with high Energy reserve and Received Signal Strength. This scheme maximizes the network lifetime by preventing early depletion of energy in the network. It is scalable and involves less energy, routing overhead, number of hops and packet losses.

\section{REFERENCES}

Amin, R., K.C. Wang and P. Ramanathan, 2007. An integrated routing and scheduling approach for persistent vehicle communication in mobile WiMAX mesh networks. Proceedings of IEEE Conference on Military Communications, Oct. 2931, IEEE Xplore Press, Orlando, FL, USA., pp: 1-7. DOI: 10.1109/MILCOM.2007.4454769

Al-Hemyari, N.K.N., A. Ismail, S. Khatun, Y.H. Tahir and Y.A. Qassem, 2008. Centralized scheduling, routing tree in WiMAX mesh networks. Proceedings of the International Conference on Innovations in Information Technology, Dec. 16-18, IEEE Xplore Press, Al Ain, pp: 539-543. DOI: 10.1109/INNOVATIONS.2008.4781702

Chen, Q. and M.C, Gursoy, 2009. Energy-efficient modulation design for reliable communication in wireless networks. Proceedings of 43rd Annual Conference on information Sciences and Systems, Mar. 18-20, IEEE Xplore Press, Baltimore, MD., pp: 811-816. DOI: 10.1109/CISS.2009.5054829 
Chen, L.W., Y.C. Tseng, Y.C. Wang, D.W. Wang and J.J. $\mathrm{Wu}, 2009$. Exploiting spectral reuse in routing, resource allocation and scheduling for IEEE 802.16 mesh networkss. IEEE Trans. Veh. Technol., 58: 301-313. DOI: 10.1109/TVT.2008.923685

De Rango, F., F. Guerriero and P. Fazio, 2012. Linkstability and energy aware routing protocol in distributed wireless networks. IEEE Trans. Parallel Distrib. Syst., 23: 713-726. DOI: 10.1109/TPDS.2010.160

El-Najjar, J., C. Assi and B. Jaumard, 2010. Joint routing and scheduling in WiMAX-based mesh networks. IEEE Trans. Wireless Commun., 9: 2371-2381. DOI: 10.1109/TWC.2010.07.091551

Jalali, R. and S. Khorsandi, 2011. Joint routing tree construction and centralized scheduling in WiMAX mesh networks. Proceedings of the 19th Iranian Conference on Electrical Engineering, May 17-19, IEEE Xplore Press, Tehran, Iran, pp: 1-6.

Jiao, Y., M. Ma, Q. Yu, K. Yi and Y. Ma, 2010. Crosslayer concurrent transmission scheduling in WiMAX mesh networks. Proceedings of the IEEE International Conference on Communication Systems, Nov. 17-19, IEEE Xplore Press, Singapore, pp: 406-410. DOI: 10.1109/ICCS.2010.5686516

Karp, B. and H.T. Kung, 2000. GPSR: Greedy perimeter stateless routing for wireless networks. Proceedings of the 6th Annual International Conference on Mobile Computing and Networking, Aug. 06-11, ACM Press, New York, USA., pp: 243-254. DOI: $10.1145 / 345910.345953$

Kelaiya, K., 2008. Routing and scheduling algorithm of IEEE 802.16 mesh backhaul network for Radio Recourse Management (RRM). Proceedings of Mobile and Pervasive Computing (PC' 08), pp: 175-179.

Kuran, M.S., G.G.T. Tugcu and F. Alagoz, 2008. Crosslayer routing-scheduling in IEEE 802.16 mesh networks. Proceedings of the 1 st International Conference on MOBILE Wireless MiddleWARE, Operating Systems and Applications, Feb. 12-15, ACM Press, Innsbruck, Austria.

Kim, M., I. Ra, J. Yoo, D. Kim and H. Kim, 2008. QoS mesh routing protocol for IEEE 802.16 based wireless mesh networks. Proceedings of the 10th International Conference on Advanced Communication Technology, Feb. 17-20, IEEE Xplore Press, Gangwon-Do, pp: 812-817. DOI: 10.1109/ICACT.2008.4493879
Lo, S.C. and L.C. Ou, 2009. Efficient algorithms for routing and centralized scheduling for IEEE 802.16 mesh networks. Proceedings of the International Conference on Scalable Computing and Communications, Sept. 25-27, IEEE Xplore Press, Dalian, pp: 212- 217. DOI: 10.1109/EmbeddedComScalCom.2009.46

Li, Y., Y. Yang and C. Cao, 2009. A novel routing algorithm in distributed IEEE 802.16 mesh networks. IEEE Commun. Lett., 13: 761-763. DOI: 10.1109/LCOMM.2009.091427

Ntlatlapa, N., 2008. A routing metric and algorithm for IEEE802.16 mesh networks. Proceedings of 3rd International Conference on Broadband Communications, Information Technology and Biomedical Applications, Nov. 23-26, IEEE Xplore Press, Gauteng, pp: 324-328. DOI: 10.1109/BROADCOM.2008.45

Molle, C., F. Peix and H. Rivano, 2008. An optimization framework for the joint routing and scheduling in Wireless Mesh Networks. Proceedings of the IEEE 19th International Symposium on Personal, Indoor and Mobile Radio Communications, Sept. 15-18, IEEE Xplore Press, Cannes, pp: 1-5. DOI: 10.1109/PIMRC.2008.4699718

Mogre, P.S., N. d'Heureuse, M. Hollick and R. Steinmetz, 2008. CORE: Centrally optimized routing extensions for the IEEE 802.16 MeSH mode. Proceedings of the 33rd IEEE Conference on Local Computer Networks, Oct. 14-17, IEEE Xplore Press, Montreal, Que, pp: 58-65. DOI: 10.1109/LCN.2008.4664152

Nahle, S. and N. Malouch, 2008. Joint routing and scheduling for maximizing fair throughput in WiMAX mesh network. Proceedings of the 19th International Symposium on Personal, Indoor and Mobile Radio Communications, Sept. 15-18, IEEE Xplore Press, Cannes, pp: 1-5. DOI: 10.1109/PIMRC.2008.4699798

Peng, L. and S. Sun, 2011. Coordinated distributed data scheduling scheme in IEEE 802.16 mesh networks. Proceedings of International Conference on Internet Computing and Information Services, Sept. 17-18, IEEE Xplore Press, Hong Kong, pp: 389-392. DOI: 10.1109/ICICIS.2011.101

Peng, M., Y. Wang and W. Wang, 2007. Cross-layer design for tree-type routing and level-based centralised scheduling in IEEE 802.16 based wireless mesh networks. IET Commun., 1: 9991006. DOI: 10.1049/iet-com:20060519 
Qassem, Y.A.A., A. Al-Hemyari, C.K. Ng, N.K. Noordin and M.F.A. Rasid, 2009. Review of network routing in IEEE 802.16 WiMAX mesh networks. Aus. J. Basic Applied Sci., 3: 3980-3996.

Tsai, T.C. and C.Y. Wang, 2007. Routing and admission control in IEEE 802.16 distributed mesh networks. Proceedings of the IFIP International Conference on Wireless and Optical Communications Networks, Jul. 2-4, IEEE Xplore Press, Singapore, pp: 1-5. DOI: 10.1109/WOCN.2007.4284179
Zhang, M., S. Wang and T. He, 2009. Study on coordinated distributed scheduling in WiMAX mesh network, Proceedings of the 5th International Conference on Wireless Communications, Networking and Mobile Computing, Sept. 24-26, IEEE Xplore Press, Beijing, pp: 1-4. DOI: 10.1109/WICOM.2009.5301219

Zhang, S., F.R. Yu and V.C.M. Leung, 2010. Joint connection admission control and routing in IEEE 802.16-based mesh networks. IEEE Trans. Wireless Commun., 9: 1370-1379. DOI: 10.1109/TWC.2010.04.081437 